\title{
Hydatid brain cyst: A delayed diagnosis in a rural setting during COVID-19
}

\author{
C J Opperman, ${ }^{1,2}$ BSc Hons (Micro), MB ChB, FC Path (SA) Micro, MMed Path (Micro); \\ J M N Enslin, ${ }^{3}$ BPhysT, MB ChB, FC Neurosurg (SA), MMed (Neurosurg); J Nuttall, ${ }^{4}$ MB ChB, FC Paed (SA), MSc (Med) (Paed); \\ A J Brink, ${ }^{1}$ MB ChB, MMed (Med Micro); S P da Fonseca, ${ }^{5}$ MB BCh; H D Tootla, ${ }^{1,6}$ MB ChB, FC Path (SA) Micro, MMed Path (Micro) \\ ${ }^{1}$ Microbiology Laboratory, National Health Laboratory Service, Groote Schuur Hospital, Cape Town, South Africa; Division of Medical \\ Microbiology, Department of Pathology, Faculty of Health Sciences, University of Cape Town, South Africa \\ ${ }^{2}$ Green Point Tuberculosis Laboratory, National Health Laboratory Service, Cape Town, South Africa \\ ${ }^{3}$ Division of Neurosurgery, Department of Surgery, Faculty of Health Sciences, University of Cape Town and Red Cross War Memorial Children's \\ Hospital, Cape Town, South Africa \\ ${ }^{4}$ Paediatric Infectious Diseases Unit, Department of Paediatrics and Child Health, Faculty of Health Sciences, University of Cape Town and Red \\ Cross War Memorial Children's Hospital, Cape Town, South Africa \\ ${ }^{5}$ Community Service Medical Officer, National Department of Health, Central Karoo District, Western Cape Province, South Africa \\ ${ }^{6}$ National Health Laboratory Service, Red Cross War Memorial Children's Hospital, Cape Town, South Africa
}

Corresponding author: C J Opperman (stefanopperman1@gmail.com)

\begin{abstract}
A previously healthy 10-year-old girl, living in a sheep-farming community in South Africa with exposure to dogs, presented to her local hospital with generalised tonic-clonic seizures. The initial clinical assessment and laboratory work-up were unremarkable. When she presented with further seizures 6 months later, attempts to arrange neuroimaging and specialist assessment were unsuccessful owing to restrictions on routine healthcare services during the SARS-CoV-2 nationwide lockdown. Subsequently, 11 months after her first presentation, she developed focal neurological signs suggestive of raised intracranial pressure. A brain computed tomography scan revealed a left-sided cerebral cyst and imminent tonsillar herniation. An emergency burr-hole procedure was performed to relieve the raised intracranial pressure, followed by definitive neurosurgical excision of cysts. Hydatid protoscolices and hooklets were seen on microscopy of cyst fluid, and treatment with albendazole and praziquantel was initiated. While her infection was treated successfully, long-term sequelae including permanent blindness and hemiparesis could potentially have been prevented with early neuroimaging and surgical intervention.
\end{abstract}

S Afr Med J 2021;111(11):1050-1054. https://doi.org/10.7196/SAMJ.2021.v111i11.16043

Hydatid disease is a neglected zoonosis caused by tapeworms in the genus Echinococcus. Cystic echinococcosis, caused by E. granulosus, is most frequently associated with human disease. ${ }^{[1]}$ It is endemic in South Africa (SA) and worldwide, but is often missed despite clues such as residence in a farming community, or exposure to dogs or farm animals. ${ }^{[2]}$ Dogs are usually the definitive host, with the tapeworm residing in the small intestine and shedding infective ova in the faeces. These infective ova are ingested by an intermediate host, such as sheep, goats or swine. The ingested ova hatch in the small intestine of the intermediate host, releasing oncospheres that penetrate the intestinal wall and migrate to various organs where they develop into cysts. The definitive host is infected after ingesting cyst-containing organs of the intermediate host. Protoscolices in these cysts evaginate in the small intestine of the definitive host and develop into the adult tapeworm, completing the life cycle. Humans are accidentally infected after ingesting infective ova, usually through exposure to dogs (Fig. 1). ${ }^{[1]}$

Hydatid cysts may occur in a single organ or in multiple organs. The liver is the most common site of infection, followed by the lungs. ${ }^{[3]}$ Other rarer sites of infection include the heart, kidneys, intestine, bone, bladder and brain. ${ }^{[4,5]}$ Brain cysts account for $1-4 \%$ of disease, with $50-75 \%$ of these cases presenting during childhood and $18 \%$ of all cases having multisite involvement. ${ }^{[6,7]}$ As such, a high index of suspicion is required to make the diagnosis of hydatid brain cysts, and timeous neuroimaging is required.
In otherwise healthy and neurologically intact individuals presenting with new-onset seizures, early neuroimaging should also be considered to exclude infective intracranial lesions, which have commonly been described as a cause for seizures in low-income countries (LICs) and low- to middle-income countries (LMICs). ${ }^{[8-10]}$ Lumbar puncture, if indicated, should also be deferred until such lesions have been excluded, owing to the risk of brain herniation from raised intracranial pressure (ICP). A noteworthy hurdle compounding exclusion of infective intracranial lesions and raised ICP is that neuroimaging and specialist care outside of major cities are sparsely distributed in LICs and LMICs, including SA. ${ }^{[11]}$ However, every effort should be made to facilitate early neuroimaging to potentially prevent devastating complications of untreated infections in the brain. Clinicians need to be aware of the differences in aetiology of seizures in LICs and LMICs v. high-income countries (HICs), and should not be misled by guidelines developed for HICs suggesting delayed or no neuroimaging for new-onset seizures. ${ }^{[12]}$

\section{Case report}

A 10-year-old girl, living in a sheep-farming community in SA and exposed to dogs, presented to her district hospital after experiencing a new-onset generalised tonic-clonic seizure. There was no history of substance abuse or head trauma, and no family history of epilepsy. Clinically she was haemodynamically stable, did not have a fever or signs of meningitis, and had no focal neurological 


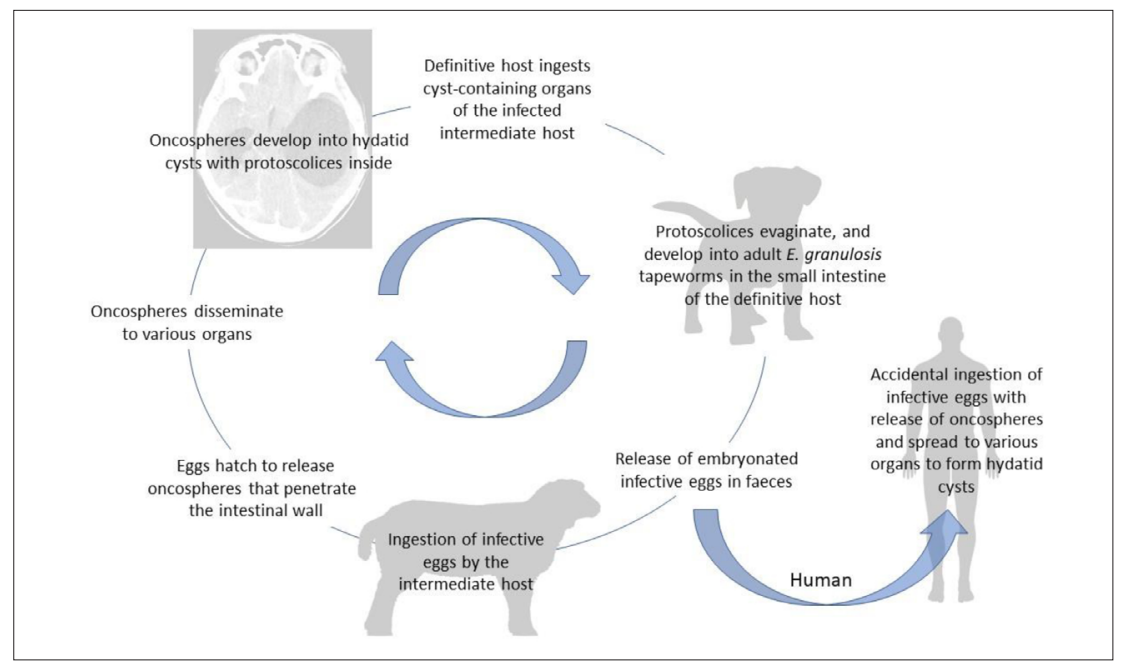

Fig. 1. Echinococcus granulosus life cycle. Dogs are usually the definitive host with the tapeworm residing in the small intestine and shedding infective ova in the faeces. These infective ova are ingested by an intermediate host, such as sheep, goats or swine. The ingested ova hatch in the small intestine of the intermediate host, releasing oncospheres that penetrate the intestinal wall and migrate to various organs where they develop into cysts. The definitive host is infected after ingesting cyst-containing organs of the intermediate host. Protoscolices in these cysts evaginate in the small intestine of the definitive host and develop into the adult tapeworm, completing the lifecycle. Humans are accidentally infected after ingesting infective ova, usually through exposure to dogs. (Figure and text modified from Centers for Disease Control and Prevention. $\left.{ }^{[1]}\right)$

deficits, developmental delay or intellectual disability. Her serum electrolytes and C-reactive protein and glucose levels were normal, and she had a slightly elevated white cell count $\left(15.94 \times 10^{9} / \mathrm{L}\right)$. Neither neuroimaging nor a lumbar puncture was performed. She presented again after another seizure 6 months later and was commenced on sodium valproate. An appointment to see a paediatrician via an outreach programme to the area was made. This local appointment, and subsequent attempts to arrange neuroimaging at the closest regional hospital, $250 \mathrm{~km}$ away, were unsuccessful owing to strict restrictions on healthcare services during the initial phases of the SARS-CoV-2 nationwide lockdown. She had multiple local follow-up appointments in her area, and paediatrician-led telephonic consultations were also done, with no new symptoms or signs of raised ICP or focal neurology noted. She continued to have breakthrough seizures almost every second month. Therapeutic sodium valproate levels were not performed. Eleven months after her index presentation she deteriorated acutely at home. She developed nausea, headaches that did not respond to analgesia, and rightsided weakness, and became increasingly lethargic and drowsy. Her family brought her to the local hospital, where right-sided weakness and an abnormal Glasgow Coma Scale score (GCS) of 10/15 were noted. She was immediately transferred to the closest regional hospital, $250 \mathrm{~km}$ away, for emergency neuroimaging and potential neurosurgical intervention.

\section{Management}

At the regional hospital, an elevated blood pressure $(155 / 63 \mathrm{mmHg})$, increased rightsided tone, a right fixed dilated pupil, a left pinpoint pupil, third cranial nerve palsy and a deteriorating GCS of 4/15 were noted. The patient was intubated and mechanically ventilated, and an emergency non-contrast computed tomography (CT) scan of the brain revealed a large left-sided frontoparietal cyst with markedly raised ICP and tonsillar herniation (Fig. 2A and B). Mannitol was administered and an emergency burr-hole procedure was done as a lifesaving measure, after discussion with the neurosurgical unit at a tertiarylevel paediatric hospital. After she had been stabilised, she was urgently transported by air to the tertiary-level paediatric hospital, $430 \mathrm{~km}$ away, for definitive neurosurgical intervention and specialist care.

At the tertiary hospital, a partially decompressed extraparenchymal hydatid cyst was noted. An emergency craniotomy with resection of the left frontoparietal cyst was performed. All residual cyst content was removed, with as little contamination as possible, although this was difficult because of adherence to underlying brain tissue. During surgery, compression of the posterior part of the temporal and parietal lobes and damage to the posterior temporal white matter were observed. Once surgical removal of the cysts was completed, the brain surface, dura and soft tissue were irrigated with hypertonic saline, followed by normal saline. Cyst fluid and tissue were sent for microbiological and histological investigation, respectively. Direct light microscopy of the cyst fluid revealed hooklets and protoscolices of Echinococcus (Fig. 3A and B), while histological examination included visualisation of the laminated membrane of the cyst wall and a single hooklet. Post-neurosurgery concerns included a posterior cerebral artery infarct, hygromas and pneumocranium, which were monitored with neuroimaging and treated conservatively without intervention. Cerebral oedema was managed with dexamethasone, from which the patient was slowly weaned over 2 weeks. Albendazole and praziquantel for 6 months were used to treat the E. granulosus infection, and sodium valproate was continued for ongoing convulsions. Chest and abdominal radiographs, abdominal ultrasound and an echocardiogram were performed to search for liver and lung cysts or cysts at other sites. These investigations were all negative. Echinococcus serology (IgG) using an enzyme-linked immunosorbent assay was negative.

\section{Outcome and follow-up}

Although the hydatid cysts were surgically removed and the patient was commenced on antiparasitic therapy, she had devastating neurological sequelae as a result of untreated complicated infection and delayed diagnosis. She initially had swallowing difficulties and aspiration, which subsequently resolved. However, her right-sided weakness remained, and she developed permanent blindness as a result of bilateral optic nerve atrophy due to sustained raised ICP. She was transferred back to her regional hospital for ongoing support and rehabilitation once she had stabilised. Marked brain improvement (Fig. 2C and D) was noted on CT scans on discharge from the tertiary paediatric hospital, and repeat neuroimaging (magnetic resonance imaging) in 3 months was scheduled.

\section{Ethical considerations}

All protected health information was removed or anonymised, and individual parent consent was obtained.

\section{Discussion}

The lifetime risk of having a seizure in the general population is high, with half occurring in childhood, and most occurring 

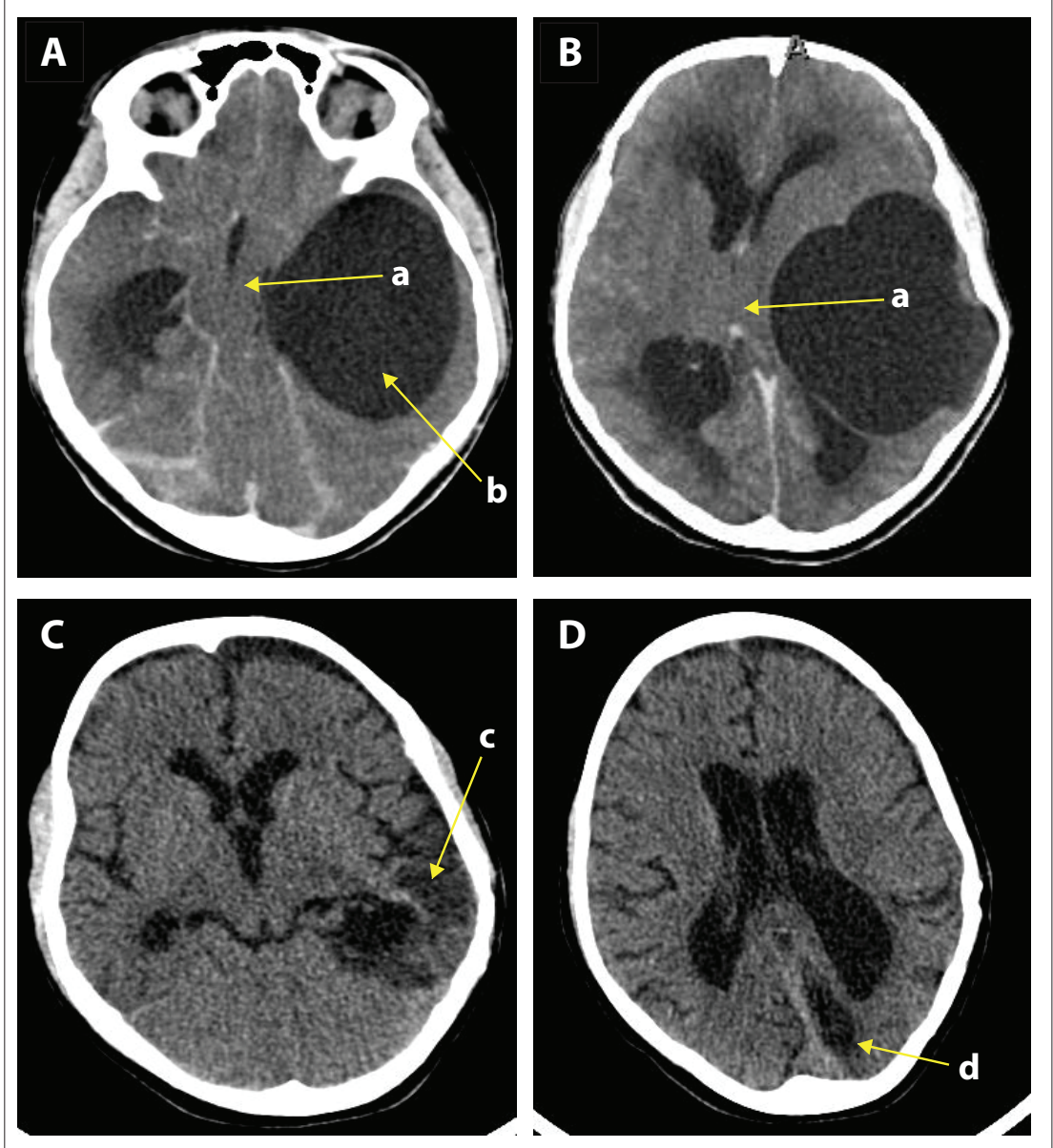

Fig. 2. (A) Post-contrast CT scan of the brain taken at the regional hospital prior to burr-hole drainage, showing a large left temporal and parietal cystic lesion with transtentorial herniation, midline shift to the right (arrow a: midline shift and herniation, arrow b: large hydatid cyst), and (B) entrapment hydrocephalus (arrow a: severe midline shift). (C) Non-contrast CT scan of the brain 3 months after surgery, showing encephalomalacia on the left parietal and temporal lobes (arrow c). The midline returned to normal, but with noticeable age-inappropriate cerebral atrophy present and (D) a left posterior cerebral artery area infarct (arrow d: encephalomalacia) related to the uncal herniation that the patient suffered prior to surgery. (CT = computed tomography.)
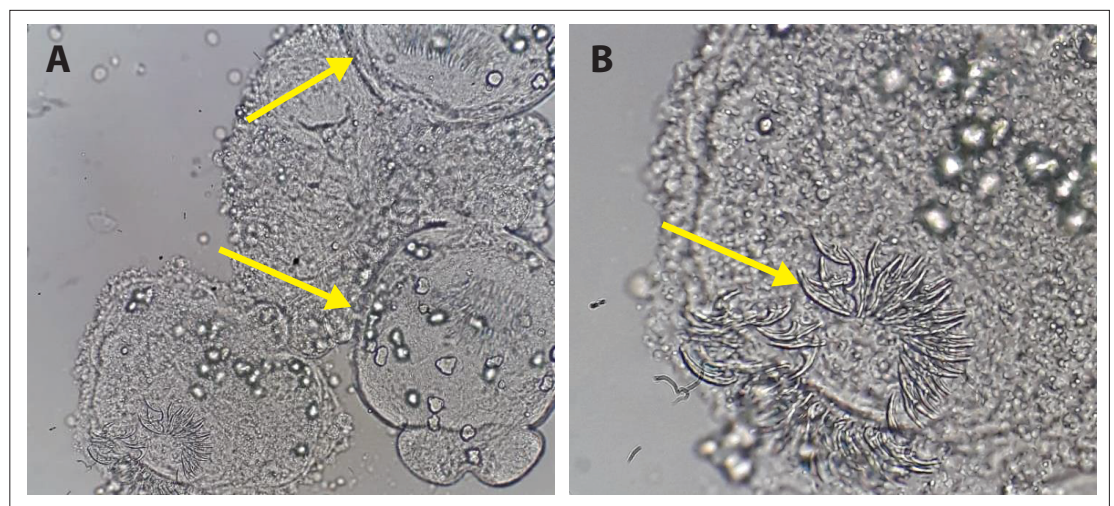

Fig. 3. Light microscopy of hydatid brain cyst fluid without stains $(A: \times 500, B: \times 1$ 000). (A) Protoscolices of Echinococcus granulosus (arrows). (B) A rostellum within a protoscolex, consisting of 28 - 50 curved hooklets (arrow) and four lateral suckers.

in developing countries. ${ }^{[8]}$ The aetiology of seizures is also different in LICs and LMICs compared with HICs, with a high burden of parasitic diseases causing new-onset seizures in otherwise healthy individuals in LICs and LMICs. ${ }^{[8-10]}$ Further clues for such zoonotic infections include residence in a farming community and exposure to dogs and farm animals. Consideration of hydatid disease should prompt early radiological intervention to prevent complications of untreated disease. Additionally, where lumbar puncture is indicated, neuroimaging should be considered first to exclude raised ICP from intracranial lesions such as neurocysticercosis or hydatid brain cysts, which could potentially lead to brain herniation and death.

Lumbar puncture was not performed in our patient, and hydatid disease was not considered as a potential cause of her seizures despite the fact that she lived in a farming community. Unfortunately, neuroimaging was not facilitated after her first seizure. After her second seizure, attempts were made to arrange neuroimaging at her closest regional hospital, $250 \mathrm{~km}$ away. These were unsuccessful owing to strict restrictions on healthcare services during the initial SARSCoV-2 nationwide lockdown. Neuroimaging was only eventually performed in this child as an emergency procedure after she presented with severe acute neurological deterioration and features of raised ICP requiring an emergency lifesaving neurosurgical intervention.

The SARS-CoV-2 pandemic, necessitating task shifting and diversion of the healthcare workforce to prioritise essential and emergency services, resulted in an unfortunate additional barrier to accessing investigations that in this case may have led to earlier medical and neurosurgical intervention and prevention of disabling neurological sequelae. This case demonstrates the need for appropriate early neuroimaging in otherwise healthy individuals who present with new-onset seizures, but specialist care and neuroimaging facilities are sparsely distributed in LICs and LMICs, and when present are often limited to major towns or cities. ${ }^{[11]}$ Accessibility, affordability, long travel distances and extended waiting times for such healthcare resources glaringly reveal the disparity between healthcare systems in well-resourced v. poorly-resourced areas.

Diagnosis of hydatid brain cysts relies on a high index of suspicion, using epidemiological clues such as exposure to dogs or farm animals, and clues from clinical presentation such as seizures, suggesting intracranial pathology. Both solid and cystic intracranial lesions may clinically present very similarly, and neuroimaging is critical to classify lesions broadly into solid or cystic. Further broad radiological traits such as tumour nodules, ring enhancement and calcification may also provide clues that support a potential diagnosis. Radiologically, hydatid cysts are non-enhancing, well- 
defined, circumscribed spherical lesions that have a thin wall with smooth margins. Calcification, surrounding oedema or rim enhancement are usually not present. In Africa, hydatid cysts should be considered for any cerebral cyst where no tumour nodule is noted on imaging, and any surgery performed should take this differential diagnosis into account, noting differences in surgical techniques required. Although perilesional oedema is not usually present with hydatid cysts, if it is present, it may indicate a secondary infection or cyst rupture. ${ }^{[13]}$ Smaller 'daughter cysts' within the main cyst can also sometimes be seen, and additional smaller cysts surrounding the main cyst can indicate prior rupture of the main cyst. Unlike liver and lung hydatid cysts, Echinococcus serology can be misleading in the presence of brain cysts and is usually falsely negative owing to minimal immune responses elicited within this privileged site. ${ }^{[3]}$ Visualisation of hydatid elements such as protoscolices (parasite larvae) and hooklets on microscopy of cyst fluid post neurosurgical intervention confirms the diagnosis.

We treated our patient with combination anti-helminth therapy using albendazole and praziquantel. Evidence supporting combination anti-hydatid therapy is mostly limited to hydatid disease in the liver, lung and peritoneum. The combination of albendazole with praziquantel has demonstrated a greater reduction in the number and/or size of cysts and increased scolicidal activity compared with albendazole alone. ${ }^{[14]}$ These factors are important to consider when managing cysts in organs such as the brain and spine, where recurrence due to spillage or cyst rupture can have devastating consequences. Praziquantel inhibits secondary cyst formation and prevents the vesicular development of protoscolices by an unknown mechanism of action. ${ }^{[15]}$ Albendazole prevents the polymerisation of tubulin into microtubules, blocks the uptake of parasite glucose and results in the depletion of its glycogen stores. ${ }^{[16]}$ The outcome is parasite starvation and inhibition of ova production in the adult cestode. The combination of these two drugs also increases the active metabolite of albendazole (albendazole sulphoxide) in the cyst fluid compared with patients receiving albendazole alone. ${ }^{[14]}$

Resection of cysts remains the main treatment modality for brain and spinal hydatid cysts, and combining surgical interventions with albendazole has also shown better outcomes for treatment of hydatid disease than surgery alone. ${ }^{[14]}$ Surgery is complicated owing to the thin wall of the cyst, which is prone to rupture. Spontaneous rupture, or iatrogenic rupture of the cyst during neurosurgical intervention, may result in dissemination with formation of secondary brain cysts or anaphylactic shock. Postoperative mortality is estimated to be $\sim 10 \%$ and increases when the cyst is ruptured during surgery. ${ }^{[6]}$ Aspiration of hydatid cysts via a burr-hole is not recommended, but the initial lifesaving procedure to relieve the intracranial pressure in our case outweighed the risk of seeding and secondary complications. Secondary infection (15\%), subdural haematoma (15\%), postoperative epilepsy (12\%) and hydrocephalus (8\%) are frequent postoperative complications, with blindness reported rarely. ${ }^{[7]}$ Our patient's blindness and posterior cerebral artery territory infarct with neurological sequelae was probably a result of brain herniation secondary to raised intracranial pressure prior to neurosurgical intervention, and could potentially have been avoided with earlier detection and intervention.

\section{Teaching points}

- Hydatid disease is endemic in SA and is often overlooked despite epidemiological clues such as residence in a farming community or exposure to dogs or other farm animals.

- Hydatid brain cysts or other intracranial infections should be considered in otherwise healthy individuals with unprovoked new-onset seizures in LICs and LMICs, and early neuroimaging is critical.

- Echinococcus serology (IgG) can be falsely negative with hydatid brain cysts. Visualisation of hydatid elements such as protoscolices (parasite larvae) and hooklets on microscopy of cyst fluid post neurosurgical intervention confirms the diagnosis.

- Management of hydatid brain cysts needs to be individualised, depending on the extent of disease and whether the cysts are anatomically amenable to neurosurgery. Ideally, cysts should be carefully resected using a surgical technique to preserve as much healthy brain tissue as possible. During surgery, it is critical to prevent rupture and spillage of cyst fluid, which can result in serious complications.

- Combination anti-helminthic therapy with albendazole and praziquantel is recommended.

- Untreated hydatid brain cysts can be life-threatening and can cause devastating and extensive neurological deficits.

\section{Conclusions}

The permanent loss of vision and profound disability in a previously healthy young girl highlight the severity of complications due to untreated intracranial infections, and in particular hydatid brain cysts. Hydatid disease is endemic in SA and yet still often not considered. Although hydatid brain cysts are relatively uncommon, new-onset seizures in an otherwise healthy and neurologically intact child should prompt consideration of infective intracranial lesions, and referral for appropriate early neuroimaging. Clues suggestive of zoonotic infection, such as residence or occupation in a farming community or exposure to dogs and other animals, are a useful tool in primary care assessment. The SARS-CoV-2 pandemic placed additional constraints on our already heavily burdened healthcare system, with a devastating outcome in this case. A strategy should be to put procedures in place to protect routine healthcare services during further waves of the SARS-CoV-2 pandemic and other unforeseen threats to the healthcare system.

\section{Declaration. None.}

Acknowledgements. We would like to thank the patient's mother, who gave her blessing and permission to write and publish this case report. In addition, we thank Kylee-Ann Baldavoo, from the National Health Laboratory Service Microbiology Laboratory, Groote Schuur Hospital, Cape Town, for taking the microscopy images. We are grateful to Dr C Tinley, from the Ophthalmology Department, Red Cross War Memorial Children's Hospital, for his review and interpretation of the ophthalmology notes. Finally, we thank the doctors at the district and regional hospitals for reviewing their clinical notes and experience with the case.

Author contributions. CJO and HDT: conceptualisation and writing of the original draft, reviewing and editing of the manuscript. JMNE, JN, AJB and SPdF: review and editing of the manuscript.

Funding. None.

Conflicts of interest. None.

1. Centers for Disease Control and Prevention. Parasites - echinococcosis life cycle. https://www.cdc.gov/ parasites/echinococcosis/biology.html (accessed 22 June 2021)

2. Ndlovu M, Thula SA, Mphahlele REM, Masekela R. Pulmonary hydatidosis: Still unrecognised in endemic regions - a 10-year review. S Afr J Child Health 2018;12(2):1-4. https://doi.org/10.7196/ sajch.2018.v12i2.1433

3. Deepak G, Gaurav J, Virendra S. A case report of primary brain hydatid cyst in a child. Iran J Neurosurg 2020;6(1):41-48. http://irjns.org/article-1-198-en.html

Sabouni F, Ferdosian F, Mamishi S, Nejat F, Monnajemzadeh M, Rezaei N. Multiple organ involvement with hydatid cysts. Iran J Parasitol 2010;5(2):65-70.

5. Polat P, Kantarci M, Alper F, Suma S, Koruyucu MB, Okur A. Hydatid disease from head to toe. Radiographics 2003;23(2):475-494. https://doi.org/10.1148/rg.232025704 
6. Alok R, Mahmoud J. Successful surgical treatment of a brain stem hydatid cyst in a child. Int J Surg Case Rep 2020;2020:5645812. https://doi.org/10.1155/2020/5645812

7. Turgut M. Intracranial hydatidosis in Turkey: Its clinical presentation, diagnostic studies, surgical Turgut M. Intracranial hydatidosis in Turkey: Its clinical presentation, diagnostic studies, surgical
management, and outcome: A review of 276 cases. Neurosurg Rev 2001;24(4):200-208. https://doi. management, and outcome:
org/10.1007/s101430100168

8. Singhi P. Infectious causes of seizures and epilepsy in the developing world. Dev Med Child Neurol 2011;53(7):600-609. https://doi.org/10.1111//.1469-8749.2011.03928.x

9. Ba-Diop A, Marin B, Druet-Cabanac M, Ngoungou EB, Newton CR, Preux PM. Epidemiology, causes, and treatment of epilepsy in sub-Saharan Africa. Lancet Neurol 2014;13(10):1029-1044. https://doi. org/10.1016/S1474-4422(14)70114-0

10. Senanayake N, Román GC. Epidemiology of epilepsy in developing countries. Bull World Health Organ 1993;71(2):247-258.

11. Magazi DS, Nkohla S, Mmako MT. Epilepsy seizure types, classification, and treatment. S Afr Fam Pract 2018;60(4):22-27.

12. Hirtz D, Ashwal S, Berg A, et al. Practice parameter: Evaluating a first nonfebrile seizure in children. Report of the quality standards subcommittee of the American Academy of Neurology, the Child Neurology Society, and the American Epilepsy Society. Neurology 2000;55(5):616-623. https://doi. org/10.1212/wnl.55.5.616
13. Taslakian B, Darwish H. Intracranial hydatid cyst: Imaging findings of a rare disease. BMJ Case Rep 2016;2016:bcr2016216570. https://doi.org/10.1136/bcr-2016-216570

14. Velasco-Tirado V, Alonso-Sardón M, Lopez-Bernus A, et al. Medical treatment of cystic echinococcosis: Systematic review and meta-analysis. BMC Infect Dis 2018;18(1):306. https://doi. org/10.1186/s12879-018-3201-y

15. Bygott JM, Chiodini PL. Praziquantel: Neglected drug? Ineffective treatment? Or therapeutic choice in cystic hydatid disease? Acta Trop 2009;111(2):95-101. https://doi.org/10.1016/j. actatropica.2009.04.006

16. Dehkordi AB, Sanei B, Yousefi M, et al. Albendazole and treatment of hydatid cyst: Review of the literature. Infect Disord Drug Targets 2019;19(2):101-104. https://doi.org/10.2174/1871526518666 180629134511

Accepted 13 September 2021. 